\title{
Féeries
}

Études sur le conte merveilleux, XVII ${ }^{\mathrm{e}} \mathrm{XIX}{ }^{\mathrm{e}}$ siècle

\section{Moralités légendaires : une fabulation de la poésie}

Legendary Moralities: An Affabulation of Poetry

\section{Henri Scepi}

\section{OpenEdition}

\section{Journals}

Édition électronique

URL : http://journals.openedition.org/feeries/1049

ISSN : 1957-7753

Éditeur

UGA Éditions/Université Grenoble Alpes

Édition imprimée

ISBN : 978-2-37747-012-9

ISSN : 1766-2842

\section{Référence électronique}

Henri Scepi, «Moralités légendaires: une fabulation de la poésie », Féeries [En ligne], 14 | 2017, mis en ligne le 31 juillet 2017, consulté le 08 septembre 2020. URL : http://journals.openedition.org/feeries/ 1049

Ce document a été généré automatiquement le 8 septembre 2020.

(c) Féeries 


\title{
Moralités légendaires : une fabulation de la poésie
}

\author{
Legendary Moralities: An Affabulation of Poetry
}

Henri Scepi

1 L'affiliation des récits des Moralités légendaires (1887) au régime générique du conte ne relève pas de l'évidence. Laforgue préfère employer le terme de "nouvelle ${ }^{1}$ ", qui présente l'avantage d'une dénomination relativement neutralisée tout en valorisant le critère distinctif de la brièveté, et celui, non moins essentiel, de la nouveauté, de l'inédit ou de l'inouï. Car les textes qu'il compose entre 1884 et 1886 - s'ils relèvent de la réécriture parodique, et $\mathrm{si}$, de ce fait, ils ne cessent de référer à des sources littéraires et à des modèles consacrés par la tradition - n'en sont pas moins révélateurs d'une entreprise d'invention. Invention d'un geste poétique, éclosion d'une manière, essor d'une voix : autant dire affirmation d'un art d'écrire en prose qui ambitionne d'être perçu et évalué comme un mode de dire sans précédent, irréductible, au risque de paraître étrange, sinon même étranger ${ }^{2}$. Il s'agit, selon la sainte loi de l'Inconscient, de ne chercher que « du nouveau, du nouveau et indéfiniment du nouveau ${ }^{3}$ ». Mais dans ce dispositif en apparence néomaniaque, le nouveau est moins la clé de voûte d'un programme concerté qu'une nécessité quasi organique.

Le projet s'inscrit de fait dans un climat d'innovation formelle, sur lequel nous reviendrons; il répond aux exigences d'une recherche expérimentale qui, après la voie lyrique empruntée par Les Complaintes (1885), s'engage simultanément sur le versant du vers libre et sur le terrain des exploits narratifs, mais en dehors des sentiers battus du réalisme et du naturalisme. Le domaine que Laforgue entreprend d'explorer en effet est ni plus ni moins celui de l'art, c'est-à-dire des manifestations esthétiques de l'idéal. L'espace de résonance que forme ce recueil se déploie et se module dans la seule dimension de l'imaginaire - ce que suggère dans un premier temps au moins l'épithète «légendaires»- devenue le point focal à partir duquel chacune des nouvelles se propose à la lecture, non pas dans une logique de type référentialiste, mais bien au contraire selon un double mouvement, de décentrement d'une part, de la réécriture par rapport au modèle, et de variation continue d'autre part, de la voix du poète par 
rapport aux scènes, aux situations, aux personnages et aux questions (philosophiques, éthiques, esthétiques) qu'il entend mettre à distance dans la perspective déformante de sa propre narration.

3 C'est à ce titre que les récits qui nous occupent peuvent être considérés comme des contes : en révélant les substrats d'un imaginaire de la création, dont certaines figures, telles que Hamlet, Pan ou Salomé, apparaissent comme les acteurs fiévreux et impatients, ils font valoir la position d'un énonciateur, narrateur bénévole ou conteur irrégulier, soucieux de faire entendre les filets ramifiés, et le plus souvent délibérément embrouillés, d'une oralité qui procède par réappropriations, détournements, reprises et pas de côté. La nouvelle se fait le lieu privilégié d'un réfléchissement de type spéculaire, et d'une réflexion, plus spéculative que pratique, sur l'art, ses visées et ses leurres. De là résulte l'habitude qu'a prise la critique laforguienne, à tort peut-être, de rabattre sur le projet des Moralités le propos programmatique contenu dans ce fragment si souvent cité : «Contes pour la jeunesse - Prendre les très populaires contes moraux, et les raconter avec une psychologie réaliste en les faisant tous rater $^{4}$.» Exemplarité paradoxale ou perversion du merveilleux, si typique de ces écritures "décadentes ${ }^{5}$ ", qui assigne au légendaire l'obligation de se défiger et de se transformer en un imaginaire plus dynamique de l'écrire et du lire, susceptible de déboucher sur une moralité d'un genre nouveau, et pour le moins déroutante.

On montrera d'abord que les enjeux du sens dans les Moralités dépendent étroitement des conditions dans lesquelles opère d'abord le défigement du légendaire, conçu comme un travail de dépoétisation et de repoétisation du fonds imaginaire qui structure la littérature et plus généralement la culture artistique. On s'attachera ensuite à l'examen des modalités fictionnelles qui dans ces récits favorisent ce que nous appellerions volontiers la "fabulation de la poésie ", une façon de raconter l'éveil à cet autre horizon de la parole, à cette autre rive du chant qui se dérobe aux mirages substantialistes de la conscience de soi et aux pièges narcissiques de l'écriture poétique. Si bien que l'ultime «merveille » vers laquelle sans doute la poésie se tourne comme par l'effet d'une giration nécessaire reste et demeure ce mythe fondateur de l'Inconscient, foyer central de l'imaginaire et matrice de toute "moralité ", dont les nouvelles de Laforgue constituent l'orchestration polytonale et bariolée.

\section{Éthique du légendaire}

5 Le recours au mythe est moins pour Laforgue une tentative de résurrection de la poésie primitive qu'une manière d'entretenir avec le passé littéraire et culturel une relation choisie et construite, vigilante et lucide ${ }^{6}$. Le propos ne vise donc pas à tirer des récits archaïques et des légendes un quelconque savoir, miraculeusement préservé et comme désenseveli du tombeau des âges; il est, tout au contraire, de soumettre tout un fonds de valeurs, de discours et de figures à une simple mais radicale question : quelle est, hic et nunc, la signification du mythe et dans quelle mesure celui-ci est-il à même de servir à l'intelligence de notre présent ? Cette question, on sait avec quelle acuité Mallarmé la pose dans son article de 1885, «Richard Wagner. Rêverie d'un poëte français ${ }^{7}$ ». Contre l'imposante domination du mythe et de son enracinement national, il préconise la neutralisation de la légende comme système de croyance, et sa promotion en tant qu'objet d'examen, défini non pas comme un langage de vérité, fondateur et unique, mais comme une structure historique, variable et multiple, comptable du jeu d'une 
«fiction», dont le «démontage », nécessairement «impie», participe du devoir suprême de la poésie ${ }^{8}$.

6 En recourant dans ses Moralités légendaires aux modèles mythiques issus de diverses époques et traditions, Laforgue revendique pour son propre compte un droit d'inventaire au nom d'un présentisme exigeant et réfléchi qui l'oblige à considérer les textes sources qu'il emploie non comme des matériaux figés, mais comme des principes actifs, aptes à inspirer une fiction moderne, une représentation du présent, en ce sens très précis où le moderne est lié au présent et, comme l'écrit Baudelaire, au plaisir que nous retirons de «sa qualité essentielle de présent ${ }^{9}$ ». Si les nouvelles de Laforgue s'écrivent à partir de "vieux canevas ", comme l'écrit Laforgue, elles sont cependant brodées «d'âmes à la mode ${ }^{10}$ ». Il s'agit bien plus d'une simple entreprise actualisante, ou d'un effort d'habillage contemporain; la visée relève d'une démarche réflexive qui prête à la reprise de type parodique un pouvoir de décentrement critique et une capacité de neutralisation des valeurs d'exemplarité véhiculées par les récits soumis à la réécriture. Le télescopage des temporalités - que soutient le ressort à loisir employé de l'anachronisme - ne donne pas lieu à une synthèse et à un dépassement résolutif ; il fait valoir un conflit, ou du moins une inadéquation stratégique qui invite à penser sur nouveaux frais les valeurs du légendaire dans leurs relations quasi organiques avec les mécanismes axiologiques de la "moralité ». Loin de redisposer d'une leçon ancestrale incessamment répétée, le légendaire apparaît ainsi comme un processus de lisibilité en cours d'élaboration: il donne moins à lire un texte déjà écrit qu'il n'invite à le recomposer, à partir de nouvelles cordonnées poétiques toujours susceptibles d'en déconstruire l'autorité et d'en afficher l'historicité. Telle est bien, en vérité, la fonction de l'humour que de maintenir l'écart entre les différents niveaux de référence et de significations ${ }^{11}$, et on comprend que l'ambition à demi avouée du Pierrot laforguien, grand maître de l'humour et de la dérision, soit de « devenir légendaire / au seuil des siècles charlatans ${ }^{12}$ ».

7 La catégorie du légendaire, si elle doit être distinguée de la conscience historique avec laquelle elle entretient une relation d'opposition ou de tension, ne peut être pensée cependant en dehors de celle-ci. Non pas que la dimension imaginative et la composante affective des activités humaines soient tout entières confiées au mythe et à ses dérivés, contre la toute-puissance de la raison positive, contre l'hégémonie du fait et de la science. Mais le romantisme - et plus particulièrement le romantisme français, comme l'a montré Claude Millet -, une fois libéré de son alliance inaugurale avec la religion, prospère grâce à un affranchissement sans cesse renouvelé de la tutelle du mythe et de la légende. «Le dispositif légendaire français vise à produire une légende qui ruine les légendes. C'est un dispositif à la fois organique et critique, fondateur et destructeur, qui fait du geste créateur un geste révolutionnaire ${ }^{13}$. » Légende unique de la pensée critique qui ne s'en laisse pas compter/conter, et qui prête aux formes et aux figures de la fable un pouvoir de rupture, une force de déliaison qui la déporte sensiblement du rayon central des normes de la croyance et des enseignements de la tradition. C'est pourquoi le légendaire, qui procède de la déconstruction des légendes et de fictions collectives, s'apparente à un efficace instrument de mise à distance morale et intellectuelle : en sapant les assises de toute croyance et de toute superstition, il suspend certes l'action dominatrice de l'esprit religieux, dont il exhibe les mécanismes captieux et les puissances d'illusion; mais il ne s'interdit nullement, en raison de la fonction critique qui l'anime, de neutraliser les prétendues vérités de l'histoire et d'en 
ressaisir par l'imagination, la rêverie ou la vision, la charge d'idéalité que les faits souvent se plaisent à contredire ou à bafouer. Telle est bien la dynamique à l'œuvre dans l'écriture de La Légende des siècles ( ${ }^{\text {re }}$ série de 1859), qui combine «l'aspect historique et l'aspect légendaire. Le second n'est pas moins vrai que le premier, souligne Hugo ; le premier n'est pas moins conjectural que le second ${ }^{14}$ ».

En somme, le légendaire ainsi conçu ressortirait à cette notion intermédiaire qu'on pourrait appeler le "possible ». Mais le possible est aussi l'autre nom de la poésie qui n'aspire ni à nier le factuel et l'historique, ni à glorifier ou à absolutiser l'imagination, mais à extraire du transitoire de l'éternel, pour le dire avec Baudelaire, ou, ce qui est une autre façon d'envisager le même phénomène, d'intérioriser, de subjectiviser l'éternel afin de le rendre aux accidents de l'existence et aux contrariétés du réel. S'il n'est pas contestable que Laforgue écrit à une époque qui voit le «dispositif légendaire » se retirer " dans la sphère privée ", s'il radicalise par là cette tendance à "l'intimisation des mythes ${ }^{15}$ propre à un certain romantisme (Nodier, Guérin, Nerval...) et à l'esthétique décadente, il accorde au légendaire une éminente valeur éthique. Car il y va tout autant, comme nous le verrons très vite, des grandes perspectives morales de l'individu - qui façonnent le prisme imaginaire du sujet poétique - que des lignes directrices de la poésie elle-même, devenue objet d'examen, matière à spéculation et à discussion, dans un espace plus nettement intersubjectif. Il paraît licite, dès lors, de concevoir la réécriture comme un processus de réhistoricisation de la légende, soumis à un double mouvement d'appropriation individuelle d'une part, et d'élargissement historique et épocal d'autre part. Raconter est à ce prix, parce que, précisément, pour Laforgue, la poésie est un jeu de forces qui affrontent, sans vraiment chercher à atteindre l'équilibre, le poids inerte du mythe et l'énergie imprédictible du présent, le légendaire défini comme héritage et l'historique pensé comme moment critique. Faire trébucher la légende, défigurer les effigies, écorner la parure des héros, tout concourt à ruiner la pureté marmoréenne de profils hiératiques au profit de la simple créature, "éphémère » et «intéressante " ${ }^{16}$. Salomé comme Andromède n'ont pas des façons de princesse ou de divinité : la première parle comme une acrobate de cabaret ( Vous allez voir ce que vous allez voir ${ }^{17}$ ), tandis que la seconde adopte le ton d'une nièce de vaudeville : «-Monstre ?... / - Bébé ? / - Dis, pourquoi tu ne m'apportes plus de pierreries, depuis quelque temps? Qu'est-ce que je t'ai fait, dis, mon oncle ${ }^{18}$ ?» Au moment même du sacrifice, Elsa a des coquetteries d'actrice et des poses sophistiquées qui ne coïncident pas avec la pureté grave de l'héroïne de Wagner. Il suffit qu'un prétendant lui tende un miroir de poche : «Et voici qu'elle se mire et s'admire ! Et au lieu de se livrer à des élégies sur le sort de ses yeux, elle arrange sa coiffure, lisse l'arc de ses célèbres sourcils, et arrange, arrange encore ses cheveux ${ }^{19}$. » Le détail relève certes de l'exercice de la parodie bouffonne et de ses variantes fumistes. Il témoigne aussi de l'inscription caractérisée de ce motif de la coiffure et de la parure dont on sait qu'il constitue depuis Baudelaire un des indices de la modernité. Dans cet intermède où elle échappe à son rôle, Elsa se révèle porteuse d'une temporalité que le mythe méconnaît. L'axe existentiel ou créaturel, comme eût dit Auerbach, confère au légendaire sa charge d'historicité, qualité qui est refusée à Syrinx, inviolablement enfermée dans ses atours de prêtresse jalouse, comme le fait observer Pan avec une malice toute contenue : «Certes, vous êtes parfaite ainsi et cette armure vous va comme un gant ${ }^{20}$. N Ne s'agit-il pas, en bref, de " vivre cette vie, quelque grands yeux étonnés qu'elle vous fasse ouvrir à chaque tournant de route ${ }^{21} »-o u$, encore, comme le dit le narrateur dans la conclusion de « Salomé » de vivre «à la bonne 
franquette ${ }^{22} »$ ? La morale peut paraître un peu courte; mais elle ne s'entend que conditionnellement, c'est-à-dire à partir et en fonction de l'opération d'invalidation critique à laquelle Laforgue soumet les contes et autres chimères pourvoyeuses d'une idéalité creuse et désincarnée.

Écran fumeux ou sublimation dévoyée, le mythe est pour Laforgue un détour qui prend le risque, à ses yeux trop grand, d'un éloignement de la vie et des principes qui interagissent dans le «tel quel de la vie ${ }^{23}$ ». Aussi, lorsque dans la nouvelle «Persée et Andromède ou le plus heureux des trois ", l'antique dragon, toujours prompt à distraire Andromède des bouffées de mélancolie qui l'obnubilent, entreprend de lui narrer un conte (Pyrame et Thisbé), celle-ci le conjure de quitter ces « histoires mortes ${ }^{24} »$. Mais " histoires mortes » et "vieillerie poétique» forment l'armature d'un discours qui s'emploie moins à corriger qu'à vouer le mythe à l'épreuve de la démythification. De là une légende, la poésie.

\section{Fabuler la poésie}

Les différents récits qui composent les Moralités légendaires peuvent être lus comme autant de tentatives de mise en ordre des données élémentaires de la création poétique. Les personnages mythiques sont tous, à un degré plus ou moins déclaré, des poètes ou des artistes. C'est bien sûr le cas de Hamlet, aquafortiste inspiré et auteur dramatique prometteur. Salomé ne manque pas non plus de talents, qui offre un petit récital à la délégation des îles Blanches Ésotériques. Pan s'arc-boute sur sa flûte à quatre notes, espérant un instrument à la hauteur de ses rêves, tandis qu'Andromède semble préférer l'ocarina et exceller, comme sa consœur Salomé, dans l'art de dire les monologues. Persée et Lohengrin exécutent avec brio des numéros de cirque ou de music-hall, comme d'autres s'emploient à " faire des ronds dans le ciel ${ }^{25}$ ». Laforgue avait bien affiché son ambition de faire « de l'art pur ${ }^{26}$ », et il convient d'entendre par là que la visée proposée, quoique abstraite, s'éclaire à l'aune d'une option exclusive qui érige l'Art en religion, c'est-à-dire d'une part en une sphère de valeurs exempte de toute connivence avec les fonctions utilitaires, didactiques ou démonstratives qu'il est toujours loisible de prêter à la poésie, et d'autre part en un système explicatif global et universel susceptible de rendre raison, in fine, des actes du vouloir et du cheminement spirituel de la pensée. Fidèle en cela à Schopenhauer, ou à son disciple Hartmann, qui voit dans la création artistique et dans le sens esthétique un miroir où vient se réfléchir en s'élucidant l'univers dans son entier, Laforgue accorde à l'art une fonction réflexive par laquelle la « volonté » entre dans le jeu de la « représentation » et s'y établit en tant qu'objet étudié ou matériau contemplé27. Une distance nécessaire favorise ainsi la bonne accommodation, et justifie la poésie comme recomposition de la vie. Une note d'un carnet de Laforgue, assez proche de l'épreuve dite de la « certitude sensible » chez Hegel, mérite ici d'être citée. «Il faut qu'une description porte la note de votre cœur, - et le moment où vous avez votre cœur, c'est non pas devant la chose crue, encombrante, mais quand plus tard, songeant, seul, nostalgique, vous évoquez l'éphémère ${ }^{28}$. Cette " poésie du souvenir » légitime une réflexivité qui ne saurait se borner aux pures stimulations de la forme et aux miroitements labiles de la surface: l'enjeu est bien plus philosophique que rhétorique. Il invite à considérer la réécriture non plus seulement comme un mécanisme inhérent aux manœuvres ordinaires de la parodie, mais aussi - et surtout - comme un geste de réappropriation de la valeur de 
la poésie, conçue comme une activité langagière et pensante, apte à révéler le fin mot de l'histoire.

11 Art pur, les Moralités donnent moins à lire une histoire de la poésie, suivie étape par étape, qu'une légende réfléchie, une fabulation concertée de l'expression poétique conçue comme l'essor, problématique et jamais vraiment accompli, d'une parole émancipée de toute tutelle, et retrempée aux sources vives d'un dire qui tourne le dos aux « codes du goût ». Laforgue met en scène dans ses nouvelles le rêve qui a toujours été le sien, et auquel, non sans une persévérante ardeur, il a voué tous ses efforts, du Sanglot de la terre aux poèmes en vers libres de La vogue: le rêve d'une langue impossible, à la fois savante et virginale, pure et raffinée. «Écrire une prose très claire, note-t-il en 1885-1886, très simple (mais en gardant toutes ses richesses) mais contournée non péniblement mais naïvement, du français d'africaine géniale, du français de Christ. Et y ajouter des images hors de notre répertoire français, tout en restant directement humaines ${ }^{29}$. $\gg$ Le propos s'enlève en effet sur fond d'invention: les personnages-artistes dont nous avons évoqué le profil entêté et capricieux ne s'abandonnent à leur fantaisie que parce que celle-ci leur offre l'occasion de se dégager des formules calcifiées et de l'empiègement régulier du langage. Mis en accusation, les mots sont comme sommés de rendre leur dernier cri, ou de s'effacer. Reprenant une célèbre sentence de son ancêtre shakespearien, Hamlet ne manque pas de rappeler, fûtce souvent hors de propos, que tout discours est vanité, toute parole promise au néant. Ainsi au fossoyeur « qui l'observe, attendant des compliments sur son arrangement des couronnes », il «aboie par la figure: Words! words! words! entendez-vous! des mots, des mots, des mots ${ }^{30} »$. Plus loin, au cours de son monologue divagant prononcé sur la sépulture de Yorick, il affirme : «Et puis, des mots, des mots, des mots! Ce sera là ma devise tant qu'on ne m'aura pas démontré que nos langues riment bien à une réalité transcendante ${ }^{31}$. "

12 C'est bien la quête, sans cesse relancée, d'une coïncidence entre le langage et l'infini - la recherche d'une «rime» absolue qui serait la garantie d'une harmonie transcendantale - qui fait de la poésie un drame, une action et un récit dont les nouvelles de Laforgue déroulent de façon plus ou moins directe et persuasive l'inlassable scénario. Hamlet, par exemple, s'enorgueillit d'échapper à la catégorie des poètes d'occasion, quoiqu'il fût contraint, comme il le confie à la comédienne Kate, de composer sa pièce révélatrice « au milieu de répugnantes préoccupations domestiques ${ }^{32}$ ». Mais la logique de l'invention poétique a pris le pas sur les desseins dénonciateurs et celui qui avait la ferme intention de "faire crier son dernier cri au sang de (son) père » se pique au jeu de l'art. «Je pris goût à l'œuvre, moi ! J'oubliai peu à peu qu'il s'agissait de mon père assassiné [...], de ma mère prostituée (vision qui m’a saccagé la Femme et m'a poussé à faire mourir de honte et de détérioration la céleste Ophélie !), de mon trône enfin ! Je m'en allais bras-dessus, bras-dessous, avec les fictions d'un beau sujet ! Car c'est un beau sujet ${ }^{33}$ !» Il est difficile de ne pas entendre dans cette confession distanciée l'aveu à peine dissimulé de l'écrivain qui s'est ingénié à retravailler Hamlet - l'œuvre et le personnage - pour donner naissance à l'insoupçonné de la poésie, à cette révélation inattendue de soi-même, qui agit comme par effraction, et qui donne à percevoir à celui qui s'y risque des gisements inépuisables de mots et d'images, de rêveries et de visions. C'est là toute "l'allégresse d'artiste » qui étreint le Hamlet de Laforgue au moment où il vit « son baptême de poète ». «Ce drame-ci, ce n'est rien, dit- 
il à Kate, [...] j'en ai encore là-haut des drames et des poèmes, des féeries et des métaphysiques, inouïs, foudroyants ou donneurs de mort lente ${ }^{34}$ !»

Plus éloquente sans doute est la « vocation » de Pan dans la nouvelle «Pan et la Syrinx, ou l'invention de la flûte à sept tuyaux ». Le titre, explicite et programmatique, invite à envisager l'histoire - forcément « légendaire » - d'une découverte. Le dieu Pan, las de son vieil instrument, ce "galoubet qui n'a que quatre notes ${ }^{35}$ » aspire à inventer une flûte plus riche et plus subtile dont les modulations seraient à la mesure des nuances et des échos d'une âme compliquée. Pour l'heure, le répertoire de son art est limité, et se borne à quelques gammes inlassablement recommencées. Quelque chose d'autre le sollicite sourdement; et il s'emploie, à grand renfort d'hymnes propitiatoires, à solliciter à son tour ce vague sentiment mêlé de mélancolie et d'optimisme qui sans cesse l'incite à «se seriner sa détresse sur son galoubet». Car "que faire, quand on aime, sinon attendre ainsi en plein air, en essayant de s'exprimer par l'art ${ }^{36}$ ? ». Pan est à l'affût de «cette révélation de temps nouveaux auxquels son génie ne va peut-être pas suffire ${ }^{37}$ ». À l'attente, toujours déçue, d'une femme, objet de ses désirs, correspond l'espoir d'un instrument, métaphore de sa voix. L'apparition de Syrinx constitue l'événement capital dans ce scénario légendaire de l'invention de la parole poétique. C'est elle, en effet, qui, en se confondant avec les eaux de la rivière, laisse filer entre les roseaux de la berge son âme éparse et musicale. De cette disparition Pan tire le bénéfice: il "jette son antique pipeau dans le tombeau de la rivière » et "donne l'accolade à ses roseaux enchantés » dont il fait « une flûte et des plus nouvelles ». Il tire de cet instrument traversé du souffle de la nymphe évanouie " une miraculeuse gamme d'ère nouvelle disant naïvement son bonheur de flûte, son bonheur de venir au monde par cette belle soirée d'Âge Pastoral ${ }^{38}$ ». D’Ovide à Mallarmé («Ces nymphes, je les veux perpétuer...»), le personnage mythique parvient ainsi à Laforgue nanti des potentialités lyriques qu'est en droit de lui reconnaître une époque de la poésie - la fin du XIX $X^{e}$ siècle - portée à célébrer ses vertus essentielles en ressaisissant les conditions symboliques de son effectuation.

\section{Merveille de l'inconscient}

14 Nul doute que les récits des Moralités ne se proposent de raconter l'histoire fantasmée d'une parole reprise à sa source: moins la genèse que le jaillissement d'un verbe revivifié, comme baigné des lueurs lustrales d'une aube du monde. Si Pan illustre exemplairement ce que Rimbaud appelle ailleurs une situation «de printemps ${ }^{39}$ », les autres textes du recueil, à des degrés divers, se penchent sur les origines d'un discours dont ils révèlent, contre l'illusion d'un lyrisme idéalisant et verbeux, toute la puissance érotico-motrice, toute la force d'exultation et d'animation vitale. Au merveilleux d'opéra-comique, dont «Lohengrin, fils de Parsifal» pourrait assez bien donner la mesure, Laforgue substitue une merveille qu'il voudrait croire nouvelle : l'essor d'une voix poétique, alliée aux mouvements secrets du désir et de l'Inconscient.

$15 \mathrm{Au}$ moment où il écrit les nouvelles qui vont former les Moralités légendaires, Laforgue traverse une période de sa création faite d'incertitudes et de remises en question ${ }^{40}$. Après Les Complaintes et L'Imitation de Notre-Dame la Lune, le projet des Fleurs de bonne volonté sitôt entrepris est à l'arrêt. Il s'avise d'une certaine inertie du discours lyrique, dont les composants sont soumis à examen, et pour certains d'entre eux neutralisés. Comme l'écrit si bien Yves Bonnefoy: «Finie l'illusion lyrique, ouvert le champ d'une 
science de la parole ${ }^{41}$.» Et c'est sans doute à la faveur d'une réflexion inlassablement poursuivie sur le versant des possibles formels de la poésie, réflexion conjointe pour ne pas dire consubstantielle à l'affermissement d'une doctrine de l'Inconscient empruntée à Hartmann, que Laforgue est amené à concevoir la nécessité d'une parole dégagée de l'emprise restrictive de la raison et de ses calculs, délestée de la tutelle de la tradition et des modèles qui la confortent en la solidifiant. Les poèmes écrits et publiés dans le courant de l'année 1886 adoptent cette allure et cette forme qu'il est aujourd'hui d'usage de qualifier de "vers libres » - dont Laforgue s'explique si clairement dans une lettre à Gustave Kahn du 10 août $1886^{42}$. Le " poème libre » témoigne de cette poussée libératrice qui fait voler en éclats les cadres esthétiques et les bordures rhétoriques, inventant une autre manière d'articulation rythmico-énonciative au sein du discours poétique, à la fois pulsation du dire et ouverture de l'écoute : il s'agit moins, en vérité, d'une explosion anarchique des codes de la poésie que d'un puissant décentrement de la perspective axiale qui jusque-là ordonnait les modèles de composition et les grilles d'intelligibilité du poème.

De cette invention, qu'elles s'emploient à intégrer, les Moralités légendaires tirent un double bénéfice. D'une part, elles se font l'écho de ce moment où la "crise de vers" rend possible une diversité déroutante de formules et de voix; d'autre part, elles recueillent et accueillent, au sein de leur agencement spécifique en prose dite narrative, des modalités rhéto-poétiques inédites qui attestent ouvertement la réorientation d'une parole tout entière déterminée par les écarts et les soubresauts d'une oralité imprédictible. Je me suis attaché à montrer ailleurs de quelle manière l'attaque de ces récits fait valoir le primat d'une voix hors norme dont les accents et les intonations tendent à supplanter le contenu de la narration, conférant ainsi aux mouvements et aux aléas de l'énonciation un relief saisissant et parfois encombrant ${ }^{43}$. Il s'agit là d'un soulignement de l'acte de narrer lui-même, dont on perçoit d'emblée le caractère factice, c'est-à-dire arbitraire, souvent convenu, toujours problématique. Après tout, cette composante orale peut bien être considérée comme une propriété du conte, lequel ne manque pas d'afficher le protocole narratif qui le soutient. Mais là où dans le conte traditionnellement ce protocole s'efface devant l'intérêt de l'histoire, qu'elle soit ou non merveilleuse, dans les Moralités de Laforgue la voix du narrateur s'entête et se reprend, trébuche ou déraille. En un mot, elle devient l'événement capital de la nouvelle. Elle donne à entendre ce « solo » qui trame la quasi-totalité des poèmes de l'année 1886.

On ne rappellera que ponctuellement la présence, au cœur même de «Lohengrin » ou de "Persée et Andromède ", de fragments textuels formellement assimilables au poème en vers libres, présence qui révèle encore une fois, en dépit des différences qui les distinguent, la proximité des deux projets, voire l'immixtion concertée de l'un dans l'autre. L'occasion « dramatique » qui les suscite est, pour le premier de ces fragments, dominée par une exaltation collective qui donne lieu à une espèce d'hymne à la gloire de la lune dans lequel il n'est pas interdit de voir une tentative de transposition de l'écriture chorale de Wagner. Ainsi, en ces « soirs de Grands Sacrifices », Notre-Dame la Lune monte à "l'horizon enchanté ». Et la voix qui accompagne cette ascension se distribue en propositions détachées (en phrases-alinéas fortement modalisées) dont le phrasé épouse celui des poèmes libres, quoiqu'elles tendent ici ostensiblement à revêtir par endroits la forme du verset :

Salve Regina des Lys!

Hostie de Léthé ! Miroir transfigurant ! 
La Mecque des Stérilités polaires!

Ô, sur l'Océan-ciboire, Eucharistie à meurtrissures, Eucharistie mal cautérisée !

Et, à l'horizon, les flots jusque-là enchantés d'accalmies, exécutent vers elle un vaet-vient berceur, ostensiblement berceur, comme la suppliant de se laisser un peu choir, ce soir, pour voir, qu'on la dorloterait si nuitamment ${ }^{44}$ ! [...]

Le deuxième fragment, celui de "Persée et Andromède", recourt à un déploiement syntaxico-rythmique à peu près comparable au précédent. Il prend également place dans le cadre d'un moment célébratif qui cette fois se consacre à « la retraite classique de l'Astre "-dont il s'emploie bien sûr à exhiber toute la facticité. Il présente cependant la caractéristique d'enchaîner les propositions en marquant par une ponctuation faible le lien interphrastique et le continu du rythme qui en dépend:

L'Astre !...

Là-bas, à l'horizon miroitant où les sirènes retiennent leur respiration,

Les échafaudages du couchant montent ;

De phares en phares, s'étagent des maçonneries de théâtre ;

Les artificiers donnent le dernier coup de main $[. . .]^{45}$.

Si l'expression se fait emphatiquement lyrique, c'est moins pour manifester une attitude émotive du sujet face au monde, que pour rendre compte de la façon dont le dire s'émeut face au langage et de quelle manière il s'actualise en exploitant les virtualités prosodiques et rythmiques qu'il mobilise (ainsi que l'illustre dans le premier extrait, non sans une note d'humour sans doute exagérément appuyée, la séquence " un peu choir, ce soir, pour voir »). Tout concourt, pour le dire autrement, à faire entendre, palpable et insistante, capricieuse et multiple, la voix de l'énonciateur, à moins que ce soit celle du poème, devenu lui-même, comme le dit Mallarmé, " énonciateur ${ }^{46}$ ». Ainsi, alors qu'il élabore des fictions de la création et de l'invention - dont «Pan et la Syrinx » pourrait constituer le modèle -, Laforgue met en scène l'émergence d'un nouveau mode d'écriture, doué d'une forte plasticité, allant du poème libre à la prose poétique, et surtout épousant l'en allé de l'inconscient, ultime merveille dont les Moralités entendent fixer la légende.

Tel est bien en effet le point nodal de cette entreprise : à la fois porter sur les fonts baptismaux une manière poétique inédite, prismatique et polymodale, et l'accorder à des fables qui ont toutes sans exception vocation à illustrer - à propos de la question récurrente de l'éternel féminin - l'indérivable religion de l'inconscient selon l'orthodoxie philosophique de Hartmann. De «Hamlet" à "Pan et la Syrinx» en passant par «Lohengrin » et "Les deux pigeons ", les nouvelles des Moralités se vouent à cette tâche inlassablement reprise : révéler les manœuvres secrètes de l'instinct, laisser affleurer les profondes et obscures menées du Désir, dont Laforgue n'ignore pas, tant s'en faut, qu'elles sont inextricablement mêlées à l'origine de la poésie et aux merveilles qu'elle est en droit de dispenser. Un court échange entre Pan et la nymphe suffit à le rappeler :

- Voyez, les hommes ne peuvent jamais être clairs devant la femme! Ils devraient faire leur déclaration en bon français, c'est-à-dire en noble et léger dialecte ionique. Non, il leur faut tout de suite la musique ! la musique si communément infinie !...

Pan se dresse furieux!

- Et vous autres! rien que le son de votre voix! Vous, tenez, la seule musique de votre voix! Est-ce plus loyal, cela? Oh! oh! misère! misère des deux côtés, en vérité ${ }^{47}$ !

21 Armée pour perpétuer l'espèce, la voix féminine n'en donne pas moins le la. Et la poésie, conçue comme le manège musical de l'infini et de l'idéal, comme l'éternelle 
fabrique du chimérique, doit s'imposer, si elle veut se survivre, un constant travail de démythification, un effort de désublimation. Par là, elle se délivre de l'illusion et s'accroît de cette dimension critique - ou seconde - qui sans cesse la dénonce ellemême comme " mirage personnel de l'univers ${ }^{48}$ » et la désigne, dans le même temps, comme seule et unique façon de saisir le sens de la vie, c'est-à-dire d'en faire la légende. La complexité de l'écriture poétique, comme le faisait observer Frye à propos de l'usage savant de la mythologie chez Joyce, «n'a pas pour but de voiler le mythe mais bien de l'expliciter. [...] Il semble que nous devons en déduire que l'intelligence érudite la plus subtile tend, tout aussi bien que le primitif et le populaire, vers un centre profond d'expériences imaginatives ${ }^{49}$ ».

\section{NOTES}

1. Voir par exemple la lettre à G. Kahn du 6 août 1885 : «Je continue mes nouvelles [...] (O.C., Lausanne, L’Âge d'homme, 1995, t.2, p. 780); ou bien la lettre du 3 juin 1886 au même destinataire : « Mon volume de nouvelles, tu en connais le principe [...]. » (Ibid., p. 852)

2. Laforgue rapporte ainsi à Théophile Ysaÿe l'appréciation du comité de la Revue illustrée à propos de sa nouvelle intitulée "Incomprise", première version sans doute des "Deux pigeons » : le comité « a trouvé que j'écrivais “comme quelqu'un qui serait étranger" (lettre de fin avril 1886, ibid., p. 845). Preuve que posséder un style, c'est, comme le notera Remy de Gourmont, "parler au milieu de la langue commune un dialecte particulier, unique et inimitable... » (« Du style et de l'écriture » [1899], dans La Culture des idées [1900], UGE, coll. «10/18», 1983, p. 23).

3. O.C., Lausanne, L'Âge d'homme, 2000, t. 3, p. 342.

4. Ibid., p. 1018.

5. Je renvoie sur cet aspect à l'ouvrage classique de référence : J. de Palacio, Les Perversions du merveilleux. Ma Mère l'Oye au tournant du siècle, Paris, Séguier, 1993.

6. On notera que par là Laforgue n'est pas wagnérien, puisque Wagner pose que «le mythe est la matière idéale du poète ", qu'il est à ce titre «le poème primitif et anonyme du peuple [...], remanié sans cesse à nouveau par les grands poètes des périodes cultivées " (Quatre poèmes d'opéra, Paris, 1860, p. XxV-XXVI). Voir Vortex, revue de l'association Jules Laforgue, « Usages du mythe ", $\mathrm{n}^{\circ} 2,2000$, Université de Liège.

7. Voir St. Mallarmé, O.C., B. Marchal (éd.), Paris, Gallimard, coll. «Bibliothèque de la Pléiade », 2003, t. 2, p. 153-159.

8. St. Mallarmé, « La Musique et les Lettres », ibid., p. 67.

9. Ch. Baudelaire, «Le Peintre de la vie moderne » [1863], O.C., C. Pichois (éd.), Paris, Gallimard, coll. «Bibliothèque de la Pléiade », 1976, t. 2, p. 684.

10. «Mon volume de nouvelles, tu en connais le principe, écrit Laforgue: de vieux canevas brodés d'âmes à la mode. » (Lettre du 3 juin 1886, O.C., ouvr. cité, t. 2, p. 852.)

11. Comme l'écrit Gilles Deleuze, l'humour est la « coextensivité » du sens et du non-sens (Logique du sens, Paris, Éditions de Minuit, 1969, p. 166). Définition qui a l'avantage de comprendre les pratiques déviantes inhérentes au rire fin-de-siècle, à commencer par le monologue moderne, qui allie l'impossible au possible. Voir D. Grojnowski, Aux commencements du rire moderne, Paris, José Corti, 1997, et notamment le chapitre « Laforgue fumiste », p. 85-98. 
12. «Locutions des Pierrots ", L'Imitation de Notre-Dame la Lune, O.C., ouvr. cité, t. 2, p. 97.

13. Cl. Millet, Le Légendaire au XIXe siècle. Poésie, mythe et vérité, Paris, PUF, 1997, p. 10.

14. V. Hugo, « Préface », La Légende des siècles, Cl. Millet (éd.), Paris, Le Livre de Poche, 2000, p. 46.

15. Cl. Millet, ouvr. cité, p. 11.

16. Je reprends ici les termes employés par Laforgue dans sa critique du système tainien du Beau idéal et des valeurs atemporelles de l'art. Voir O.C., ouvr. cité, t. 3, p. 359 et suiv.

17. J. Laforgue, «Salomé », Moralités légendaires, D. Grojnowski et H. Scepi (éds), Paris, Flammarion, 2000, p. 147.

18. « Persée et Andromède, ou le plus heureux des trois », ibid., p. 159.

19. « Lohengrin, fils de Parsifal », ibid., p. 115.

20. «Pan et la Syrinx, ou l'invention de la flûte à sept tuyaux », ibid., p. 197.

21. «Persée et Andromède, ou le plus heureux des trois », ibid., p. 176.

22. «Salomé », ibid., p. 153.

23. Note à "L'Art moderne en Allemagne ", O.C., ouvr. cité, t. 3, p. 349.

24. "Persée et Andromède, ou le plus heureux des trois ", ibid., p. 160.

25. « Hamlet ou les suites de la piété filiale », ibid., p. 59.

26. Lettre à T. Ysaÿe, de juillet 1885, O.C., ouvr. cité, t. 2, p. 774.

27. Voir sur ce point «L'Art moderne en Allemagne », O.C., ouvr. cité, t. 3, p. 339 et suiv.

28. Carnet 1884-1885, O.C., ouvr. cité, t. 2, p. 976.

29. O.C., ouvr. cité, t. 3, p. 1054.

30. « Hamlet ou les suites de la piété filiale », O.C., ouvr. cité, t. 3, p. 74.

31. Ibid., p. 76.

32. Ibid., p. 83.

33. Ibid., p. 63.

34. Ibid., p. 83.

35. «Pan et la Syrinx, ou l'invention de la flûte à sept tuyaux ", dans ouvr. cité, p. 181.

36. Ibid.

37. Ibid., p. 198.

38. Ibid., p. 199.

39. Lettre à Théodore de Banville, du 24 mai 1870, O.C., A. Guyaux (éd.), Paris, Gallimard, coll. «Bibliothèque de la Pléiade ", 2009, p. 323.

40. À Kahn le 21 mars 1886, il confie : «Je n'écris rien, ne lis guère, ne pense et ne projette rien d'art, et trouve cela un état naturel. » (O.C., ouvr. cité, t. 2, p. 828.)

41. "Hamlet et la couleur", dans J. Bem, J.-P. Bertrand, Y. Bonnefoy et al., Laforgue aujourd'hui, textes réunis et présentés par J. Hiddleston, Paris, José Corti, 1988, p. 175.

42. Voir O.C., ouvr. cité, t. 2, p. 862.

43. Voir H. Scepi, "L'initiale textuelle dans les Moralités légendaires ", L'Incipit, Revue La Licorne, L. Louvel (dir.), Université de Poitiers, 1997.

44. «Lohengrin, fils de Parsifal », dans ouvr. cité, p. 112.

45. «Persée et Andromède, ou le plus heureux des trois », dans ouvr. cité, p. 168.

46. «Crise de vers ", O.C., ouvr. cité, t. 2, p. 209.

47. «Pan et la Syrinx, ou l'invention de la flûte à sept tuyaux », dans ouvr. cité, p. 188.

48. «L'Art moderne en Allemagne », O.C., ouvr. cité, t. 3, p. 361.

49. N. Frye, Anatomie de la critique, trad. G. Durand, Paris, Gallimard, 1969, p. 146. 


\section{RÉSUMÉS}

Cet article se propose de montrer que les enjeux du sens dans les Moralités dépendent étroitement des conditions dans lesquelles opère d'abord le défigement du légendaire, conçu comme un travail de dépoétisation et de repoétisation du fonds imaginaire qui structure la littérature et plus généralement la culture artistique. Les modalités fictionnelles mises en œuvre dans ces récits favorisent une "fabulation de la poésie », qui en mettant en scène des personnages artistes aux prises avec les aléas de leur vocation, se dérobe aux mirages substantialistes et aux pièges narcissiques de l'écriture poétique. D'où il ressort que l'ultime "merveille " vers laquelle en dernière analyse la poésie se tourne, comme sous l'effet d'une aimantation nécessaire, reste et demeure ce mythe fondateur de l'Inconscient, foyer central de l'imaginaire et matrice de toute «moralité », dont les nouvelles de Laforgue proposent au gré de leurs variations l'orchestration fantaisiste et fantasmée.

This article aims to show that the stakes of meaning in Laforgue's Moralités légendaires depend closely on the conditions under which the legendary defiance first works, as it is conceived as a work of depoetization and repoetization of the imaginary fund that structures literature and more generally artistic culture. The fictional modalities used in these narratives favor a "fabulation of poetry", which, by depicting artist figures struggling with the vagaries of their vocation, evades philosophical mirages and the narcissistic traps of poetic writing. As a consequence, it emerges that the ultimate "wonder" towards which poetry turns in the final analysis as if it were a necessary magnetization, remains this founding myth of the Unconscious, the central focus of the imaginary and the matrix of all "morality", of which the narratives of Laforgue propose a fanciful and fantasized orchestration.

\section{INDEX}

Mots-clés : Jules Laforgue, Moralités légendaires, contes poétiques en prose, le légendaire, dépoétisation, repoétisation, fabulation de la poésie, Hartmann, Inconscient, imaginaire

\section{AUTEUR}

HENRI SCEPI

Université Sorbonne Nouvelle - Paris 3 